\title{
Bedsharing and Breastfeeding: The Academy of Breastfeeding Medicine Protocol \#6, Revision 2019
}

\author{
Peter S. Blair, ${ }^{1}$ Helen L. Ball, ${ }^{2}$ James J. McKenna, ${ }^{3,4}$ Lori Feldman-Winter, ${ }^{5}$ Kathleen A. Marinelli, \\ Melissa C. Bartick, ${ }^{8}$ and the Academy of Breastfeeding Medicine
}

\begin{abstract}
A central goal of the Academy of Breastfeeding Medicine is the development of clinical protocols for managing common medical problems that may impact breastfeeding success. These protocols serve only as guidelines for the care of breastfeeding mothers and infants and do not delineate an exclusive course of treatment or serve as standards of medical care. Variations in treatment may be appropriate according to the needs of an individual patient.
\end{abstract}

\section{Purpose}

B EDSHARING PROMOTES BREASTFEEDING INITIATION, ${ }^{1}$ duration, ${ }^{2-7}$ and exclusivity. ${ }^{7,8}$ Medical and public health organizations in some countries recommend against bedsharing, citing concerns over increased risk of sleeprelated infant death. ${ }^{9,10}$ However, bedsharing may only be a risk in hazardous circumstances as demonstrated by epidemiological study (Table 1$).{ }^{11}$ We aim to clarify the currently available evidence regarding the benefits and risks of bedsharing, and offer evidence-based recommendations that promote infant and maternal health through increased breastfeeding duration. ${ }^{12}$ The recommendations in this protocol apply to mother-infant dyads who have initiated breastfeeding and are in home settings, and are not intended for use in hospitals or birth centers.

\section{Summary and Recommendations}

\section{Summary}

Levels of evidence (1-5) from the Oxford Centre for Evidence Based Medicine ${ }^{13}$ are listed in parentheses, and are based on the citations as described below in the supporting material. See the supporting material for the ways in which we define "bedsharing," "SIDS," and "separate sleep" for purposes of this protocol. "Breastsleeping" is defined there as well.

Overall, the research conducted to date on bedsharing and breastfeeding indicates that nighttime proximity facilitates breastfeeding duration and exclusivity (levels 2-3). ${ }^{2,4,14}$ Discussions about safe bedsharing should be incorporated into guidelines for pregnancy and postnatal care. ${ }^{15-19} \mathrm{Ex}-$ isting evidence does not support the conclusion that bedsharing among breastfeeding infants (i.e., breastsleeping) causes sudden infant death syndrome (SIDS) in the absence of known hazards (level 3) (see Table 1). ${ }^{11}$ Larger studies with appropriate controls are needed to understand the relationship between bedsharing and infant deaths in the absence of known hazards at different ages. Not all hazards are individually modifiable after birth (e.g., prematurity). Accidental suffocation death is extremely rare among bedsharing breastfeeding infants in the absence of hazardous circumstances (levels 2-3), ${ }^{20}$ and must be weighed against the consequences of separate sleep. There are consequences to breastfeeding with separate sleep (even with room-sharing) that include the risk of early weaning, the risk of compromise to milk supply from less frequent nighttime breastfeeding, and unintentional bedsharing (levels 1-3). ${ }^{5,21,22}$ Recommendations concerning bedsharing must take into account the mother's

\footnotetext{
${ }^{1}$ Centre for Academic Child Health, University of Bristol, Bristol, United Kingdom.

${ }^{2}$ Infancy and Sleep Centre, Department of Anthropology, Durham University, Durham, United Kingdom.

${ }^{3}$ Department of Anthropology, Santa Clara University, Santa Clara, California.

${ }^{4}$ Mother-Baby Sleep Lab, Department of Anthropology, University of Notre Dame, South Bend, Indiana.

${ }_{5}^{5}$ Department of Pediatrics, Division of Adolescent Medicine, Cooper Medical School of Rowan University, Camden, New Jersey.

${ }^{6}$ Department of Pediatrics, University of Connecticut School of Medicine, Farmington, Connecticut.

${ }^{7}$ Connecticut Children's Medical Center, Division of Neonatology, Hartford, Connecticut.

${ }^{8}$ Department of Medicine, Cambridge Health Alliance and Harvard Medical School, Cambridge Massachusetts.
} 
TABle 1. Hazardous Risk Factors or Circumstances During Bedsharing

These are factors that increase the risk of SIDS and fatal sleeping accidents, either alone or when combined with bedsharing. $11,26,41,42$

- Sharing a sofa with a sleeping adult ("sofa-sharing")

- Infant sleeping next to an adult who is impaired by alcohol $^{\mathrm{a}}$ or drugs

- Infant sleeping next to an adult who smokes

- Sleeping in the prone position

- Never initiating breastfeeding

- Sharing a chair with a sleeping adult

- Sleeping on soft bedding

- Being born preterm or of low birth weight

${ }^{a}$ Amounts of alcohol causing impairment are discussed in the text. SIDS, sudden infant death syndrome.

knowledge, beliefs, and preferences and acknowledge the known benefits as well as the risks (level 5). ${ }^{23,24}$

\section{Recommendations}

All families should be counseled about safe sleep. Table 2 summarizes safe sleep advice in order of importance based on the strength of the evidence. In addition, we recommend the following:

1. Discussion with open-ended questions from health care providers concerning bedsharing safety should happen with all parents, as bedsharing is likely to happen whether intended or not (level 4). ${ }^{25}$ These discussions should take place early in the perinatal course and continuously throughout infancy, and include as many caregivers as

Table 2. Elements of Safe Bedsharing Advice, IN ORDER OF IMPORTANCE

1. Never sleep with infants on a sofa, armchair, or unsuitable surface, including a pillow (level 3 ). ${ }^{11}$

2. Place infants to sleep away from any person impaired by alcohol or drugs (level 3). ${ }^{11}$

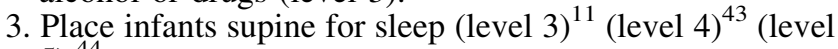
$5)^{44}$

4. Place infants to sleep away from secondhand smoke and away from a caregiver who routinely smokes (level 1$)^{28}$ and clothing or objects that smell of smoke (thirdhand smoke) (level 5). ${ }^{45}$ (In cases where the mother smokes, this will not be possible).

5. The bed should be away from walls and furniture to prevent wedging of the infant's head or body (level 1). ${ }^{46}$

6. The bed's surface should be firm, just as with a crib (level $3),{ }^{41}$ without thick covers (e.g., duvets, doonas), pillows, or other objects that could cause accidental head covering and asphyxiation.

7. The infant should not be left alone on an adult bed (level 1). ${ }^{47}$

8. Adoption of the C-position ("cuddle curl"), with the infant's head across from the adult's breast, adult's legs and $\operatorname{arm}(\mathrm{s})$ curled around the infant, infant on their back, away from the pillow, is the optimal safe sleeping position (Fig. 1) (level 4). ${ }^{48,49}$

9. There is insufficient evidence to make recommendations on multiple bedsharers or the position of the infant in bed with respect to both parents in the absence of hazardous circumstances. ${ }^{50,51}$ Each locality should consider the cultural circumstances unique to its situation with respect to sleep conditions.
Table 3. Risk Minimization Strategies for Families in Which Bedsharing Is High RisK

Increased promotion and support of breastfeeding (level 1) ${ }^{12,42,52}$ (level 3). ${ }^{52}$

Referral for smoking cessation and alcohol and/or drug treatment (level 1) ${ }^{28}$ (level 3). ${ }^{11}$

Enhanced repeated multimodal messaging regarding risks of sofa-sharing, bedsharing where hazardous factors are present, including sleeping next to an impaired adult and smoke avoidance. Text messaging and e-mail, including use of video and social media may be helpful if available to parents (level 2). ${ }^{34,53,54}$

Sidecars or in-bed devices (e.g., Pēpi-Pod ${ }^{\circledR}$; wahakura) can be considered (level 2). ${ }^{1,55}$

Emphasize room-sharing where and when bedsharing cannot be done safely.

Take into account the importance of the partner and other support persons' involvement in the infants' sleep time activities.

possible. Open-ended questions that have been found to be successful in opening conversations include:

a. "What are your plans for where your baby will sleep?"

b. "What does that sleep area look like?"

c. "Does your baby ever end up in bed with you?"

2. Screen families at increased risk of infant death with bedsharing: infants who were born preterm (level 2$)^{26}$ (level 3), ${ }^{27}$ exposed to tobacco antenatally (level 1) ${ }^{28}$ $\left(\right.$ level 4) ${ }^{29}$ (level 5), ${ }^{30}$ live with smokers (level 1) ${ }^{28}$ (level 3) ${ }^{11}$ (level 4), ${ }^{31,32}$ and those who live with people who consume alcohol (level 3$)^{11}$ or drugs and, therefore, might be in charge of an infant and could fall asleep with the infant.

3. Information and counseling about safe bedsharing should be provided even to those parents for whom bedsharing should be discouraged (those with hazardous conditions or circumstances), as one must assume that parents may bedshare anyway, even if unintentionally (level 1). ${ }^{33}$ See Table 3 for risk minimization strategies.

a. These discussions can include how to make sleep areas as safe as possible, and can reflect how to minimize hazardous circumstances, even if they are not eliminated (See Table 2).

b. For instance, if a parent who smokes is bedsharing, breastfeeding, sleep positioning, sleep surface, bedding, and where infant naps when alone can all be discussed.

Conversations when a family is bedsharing should be nonjudgmental and acknowledge context.

- Ending stigma around bedsharing and educating all parents about safe bedsharing have the potential to reduce infant deaths. Bedsharing evolved from innate human biological and behavioral mechanisms. It is not a singular, discrete, or coherent practice, but is composed of a diverse range of behaviors, some of which may carry risks, making it particularly important to discuss bedsharing safety.

- Discussing the concept of breastsleeping with breastfeeding parents allows a way to discuss safe bedsharing 
in this context. Using the theory of planned behavior, counseling about safe breastsleeping is most likely to be effective if it is consistent with both social norms and attitudes (level 2). ${ }^{34}$

- Scripting tools are important resources. ${ }^{35,36}$ Beginning with an open-ended inquiry helps to identify an understanding of patients' and families' lived experiences. It is critical to recognize that evidence-based medicine integrates "compassionate use of individual patients' predicaments, rights, and preferences" (level 5). ${ }^{23}$ Nonjudgmental counseling helps to build trusting patientprofessional relationships for both disclosure and effective counseling (level 5). ${ }^{10}$

Public policy recommendations. Structural societal interventions are essential interventions regardless of counseling on sleeping arrangements. As many parents will have limited contact with the health care system, ${ }^{33}$ advocating for structural changes is critical. Policymakers should address the following strategies that may lower infant mortality:

1. Increasing tobacco prices, a strategy associated with an immediate marked decrease in infant mortality in Europe (level 5). ${ }^{37}$

2. Ending racial bias in the health care system that undermines breastfeeding and leads to poor maternal and infant outcomes(level 2) ${ }^{38,39}$ (level 3). ${ }^{40}$

3. Advocating for the allocation of research funding and resources focusing on the risk factors for sleep-related infant death commensurate with the evidence-based level of risk.

\section{Evidence Base and Supporting Material}

\section{Definitions}

SIDS is defined as the sudden death of an infant that is unexplained after a case review and/or autopsy and death scene investigation have been performed. SIDS has a specific code, R95, under the International Classification of Diseases, 10th Revision (ICD-10). ${ }^{56}$ The code for "other ill-defined and unspecified causes of death" (R99) is used when the cause of death is unknown or there is inadequate evidence to classify as SIDS, as when SIDS is suspected but a full investigation has not been performed. Accidental suffocation or strangulation in bed (ASSB, W75) is coded when the death was due to asphyxia, strangulation, or suffocation, in a bed, crib, sofa, or armchair.

Sudden unexpected infant death (SUID), also known as sudden unexpected death in infancy (SUDI), is an overarching term for all unexpected deaths, both those that remain unexplained (coded as R95 or R99) and those in which a full causal explanation is eventually found.

Proxy measure for SIDS: For any international comparisons for the purpose of this protocol, we use a proxy measure for SIDS by adding deaths assigned to these three codes together (R95 + R99 + W75) as a composite measure of unexplained SUDI (or SUID) ${ }^{57}$ This is due to our recognition of the diagnostic shift pointed out by Taylor et al. ${ }^{58}$ and ShapiroMendoza et al. ${ }^{59}$ over the past decade in which some pathologists and medical examiners seem reluctant to use SIDS (R95) because the diagnosis requires the exclusion of any other cause of death. Therefore, the use of codes R99 or W75 is preferred, despite incomplete or minimal evidence that overlaying (accidental smothering) may be the causal factor. Diagnostic shift toward ASSB (W75) is more common in the United States than in the United Kingdom. ${ }^{60}$

Bedsharing is defined as an infant sharing an adult bed with an adult for sleep, and for this protocol we are defining this as with the infant sleeping next to a caregiver, most often the mother. The bed may consist of a mattress or futon with varying levels of firmness depending on the filling.

Cosleeping is a term that may include both sleeping on a shared surface and sleeping in proximity, but not necessarily on a shared sleep surface. For clarity, "cosleeping" is not used in this protocol.

Separate versus solitary sleep: We refer to "separate sleep" as room-sharing without bedsharing, whereas "solitary sleep" refers to sleeping in a separate room from parents.

\section{History, context, and anthropology of infant sleep location}

Human milk, lower in solute compared with milk of other species (e.g., bovine) ${ }^{61}$ is digested very quickly. The rapidly growing infant breastfeeds at least $8-12$ times in 24 hours. ${ }^{62}$ Frequent feeding is difficult if the infant is not in close contact with his or her breastfeeding mother day and night. ${ }^{1,8}$ Parentinfant bedsharing with breastfeeding constitutes the human evolutionary norm as demonstrated in anthropological research. $^{63-67}$

In industrialized countries until the early 20th century, most infants were bedsharing and breastfeeding. ${ }^{68}$ After that time, solitary sleep developed as an ideal among the middle classes, reinforced by the growing trends of artificial feeding and medicalization of childbirth, separating infants from mothers. ${ }^{65,68}$ Sleep training also became increasingly popular in some industrialized societies. ${ }^{68}$ Human milk substitutes (e.g., infant formula) helped this trend, as infants who receive them tend to feed less frequently ${ }^{69}$ and may sleep more deeply than breastfed infants. ${ }^{70}$

Concerns about infant sleep duration and location did not appear until after the late 19th and early 20th centuries in industrialized countries, ${ }^{68}$ indicating that infant sleep research has taken place within an historical context in which feeding of human milk substitutes and solitary sleep promotion were normative. Although parents and caregivers in the majority of cultures sleep in proximity to their infants, organizations in some countries, including the United States, Canada, and Germany, recommend that even breastfeeding mothers should never share a sleep surface with their infants. ${ }^{10,71-73}$

The concept of "breastsleeping" was proposed to describe a biologically based model of sustained contact between the mother and infant, starting immediately after birth, in which sleeping and breastfeeding are inextricably combined, assuming no hazardous risk factors. ${ }^{15,64}$ Described in cultures around the world, the breastsleeping mother and infant feed frequently during the night while lying in bed together, and by morning, the mother may not recall how many times she fed or for how long. ${ }^{74}$ The breastsleeping concept acknowledges the critical role that immediate and sustained maternal contact plays in helping establish optimal breastfeeding $^{63,75,76}$; and recognizes that the behavior and physiology of breastsleeping dyads may be different from that of 


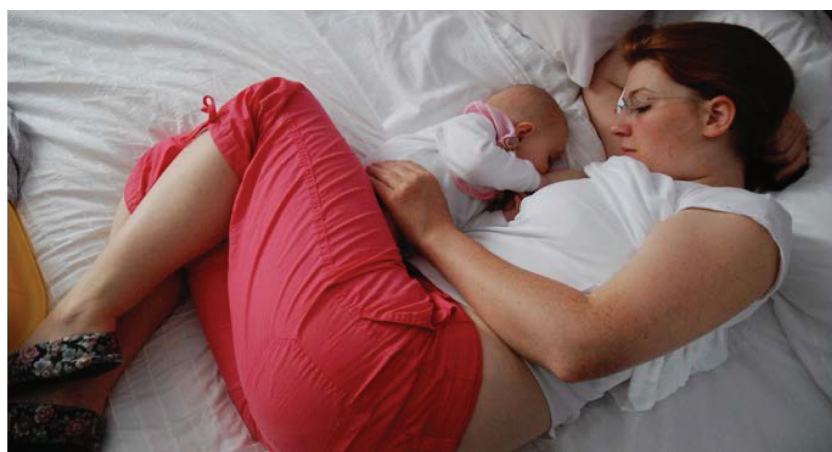

FIG. 1. C-position or "Cuddle curl." Breastsleeping mothers adopt a characteristic position (Protective $\mathrm{C}$ or Cuddle Curl) in which they make a safe space for their baby to sleep with their bodies. Mother's arm is above the baby's head preventing him/her moving up the bed into the pillows, and her knees are tucked under his/her feet to prevent him/her moving down the bed. Baby is positioned flat on his/her back on the flat mattress for sleep, and next to the mother's breasts for easy feeding. (C) Baby Sleep Information Source, licensed for use under Creative Commons, 2016. Color images are available online.

bedsharing nonbreastfeeding dyads, signifying that the safety assessments for bedsharing with breastfeeding versus feeding human milk substitutes likely require different approaches. $4,21,77,78$

When breastfeeding mothers sleep with their infants, they protect them from potential physiological stressors including airway covering and overheating by their characteristic sleep position (curled around their infants, making a constrained sleep space with their bodies), known as the C-position ${ }^{48}$ or "cuddle curl",49 (Fig. 1). Their continued vigilance through microarousals prompts regular infant arousals throughout the night. ${ }^{21,78-80}$ In two small video studies, mothers who had never breastfed were observed to exhibit these protective behaviors less frequently. ${ }^{78,81}$

Compared with breastfeeding infants who sleep alone, breastsleeping infants spend less time in stages 3-4 (deep) sleep, and more time in stages 1-2 (lighter) sleep, facilitating rapid infant awakening and termination of apneas. Additional time nurturing through breastsleeping, as compared with less time nurturing when sleeping separately, may affect epigenetic responses to stress in the infant through the possible influence of maternal care on infant regulatory responses. ${ }^{82}$

Despite decades of advice to avoid mother-infant sleep contact, researchers report that on any given night, 20-25\% of U.S. and U.K. infants $<3$ months of age share a bed with a parent for sleep at least some of the nights, ${ }^{83,84}$ and $>40 \%$ of infants in Western* societies, in general, do so at some point in the first 3 months. ${ }^{83,85-91}$ These proportions may be underreported due to the stigma associated with bedsharing, especially in the United States. Parents express various reasons for sleeping with their infant, including deeply rooted cultural or religious beliefs and parenting philosophies, physiological links between lactation and nighttime

*We understand "Western" as an ideological construct, not a geographic one.

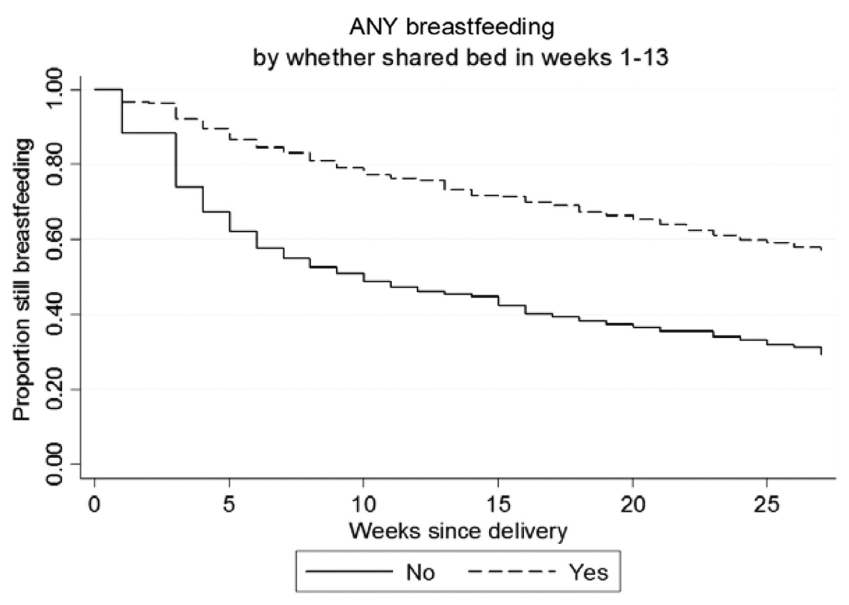

FIG. 2. Bedsharing is strongly associated with breastfeeding continuation. Source: Ball et al., 2016.

breastfeeding, and a biological compulsion that drives the urge for close contact. ${ }^{25,89,92-94}$ They explain that sleeping with their infant makes nighttime care easier, helps them monitor their infant, provides comfort, and still allows them to sleep. ${ }^{8,25,95}$ Sometimes parents report having nowhere else to put their infant at night, or that they have fallen asleep with their infant unintentionally. ${ }^{25,89,96}$ Others report bedsharing in response to either the mother or her infant being deaf, to keep infants safe from environmental harms (i.e., vermin, gunfire, and earthquakes) and to protect them from SIDS. ${ }^{97-99}$

Breastfeeding mothers comprise the largest group of bedsharers. ${ }^{92}$ Sleep contact between mother and infant facilitates nighttime breastfeeding, with multiple studies demonstrating that bedsharing is associated with more frequent nighttime feeds (promoting milk production), and more months of breastfeeding ${ }^{2,4,14,90}$ (Fig. 2). Those women with the strongest prenatal intention to breastfeed are more likely to bedshare, ${ }^{4}$ whereas breastfeeding women who did not initially intend to bedshare often end up doing so. ${ }^{8,100}$ Although bedsharing breastfeeding mothers wake frequently to feed, they are awake for shorter periods and fall back to sleep more rapidly. ${ }^{79}$ Thus they achieve greater sleep duration $^{101}$ than nonbedsharing mothers. ${ }^{14}$ Bedsharing is a strategy used by breastfeeding mothers to reduce physical and social costs, for example, sleep disruption. ${ }^{102}$ One observational study has shown that, compared with mothers who room-shared without bedsharing, mothers who bedshared were more likely to report exclusive breastfeeding (adjusted odds ratio [OR]: 2.46; 95\% confidence interval [CI]: 1.763.45 ) or partial breastfeeding (adjusted OR: 1.75; 95\% CI: 1.33-2.31)..$^{7,103}$ Therefore, advice to avoid bedsharing has the potential to undermine breastfeeding goals, $, 3,8,104,105$ and may increase risk of sleeping in unsafe environments such as sofas. 106

Although mothers and infants can sleep apart and still breastfeed exclusively, doing so results in fewer sessions of breastfeeding per night: bedsharers have double or triple the number of breastfeeding sessions and total amount of breastfeeding time compared with solitary sleepers. ${ }^{6}$ Fewer than half as many feedings occurred for mothers whose infants slept alone in a bedside bassinet (Fig. 3a) in the postnatal ward 
FIG. 3. Bassinet, sidecar, wahakura, and Pēpi-Pod $\left.{ }^{(}\right)$. (a) bassinet, (b) sidecar, (c) wahakura, (d) Pēpi-Pod. Color images are available online.

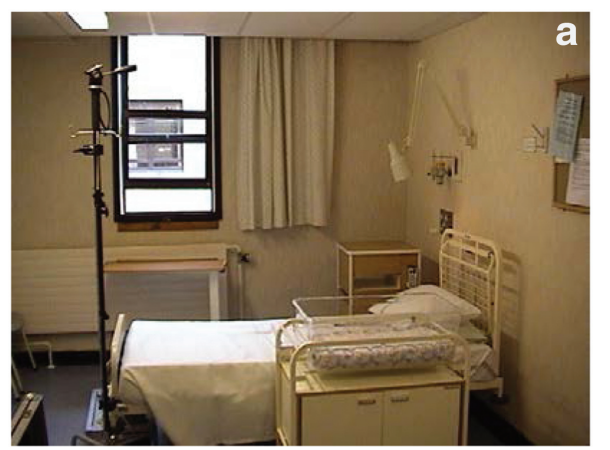

Bassinet

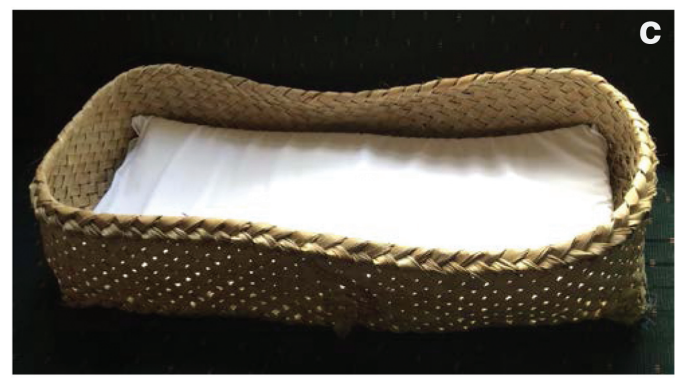

Wahakura

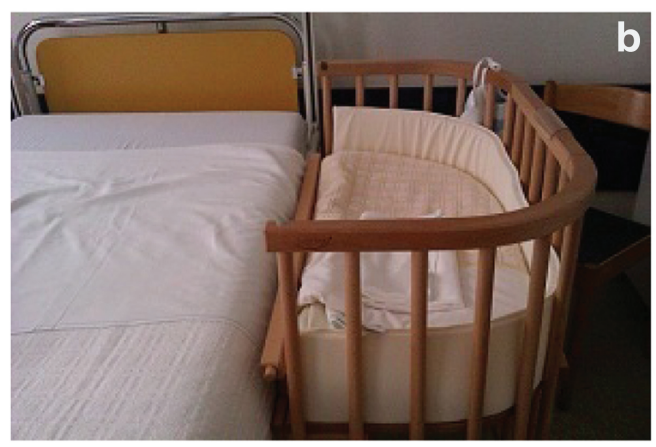

Sidecar compared with a sidecar (Fig. 3b) or with bedsharing in a randomized trial examining breastfeeding initiation. ${ }^{1}$ In one study among a population with low breastfeeding rates, advice to room-share without bedsharing achieved a similar duration of "any" breastfeeding, but not exclusive breastfeeding. ${ }^{22}$

\section{Bedsharing and SIDS:}

\section{epidemiological/observational evidence}

Feeding of human milk substitutes (formula) is associated with a markedly increased risk of SIDS. ${ }^{42}$ This may be due to lower infant arousal thresholds and increased infection risks compared with breastfeeding dyads. ${ }^{52}$ In addition, videographic studies show that breastsleeping infants consistently sleep with their heads well below pillows as they are positioned near their mother's breasts, ${ }^{21,78,81}$ which might lower suffocation risk, in contrast to infants who are fed human milk substitutes, who have been noted to be placed intentionally on or around pillows. Videographic data show that breastsleeping infants rarely sleep prone. ${ }^{21,81}$ Hauck et al. made the case that breastfeeding is causally associated with a reduction in SIDS based on biologic plausibility, consistency of findings, strength of association (which has since gotten stronger ${ }^{42}$ ), timing of association, and dose-response effect, and is not merely a marker for other protective factors including absence of tobacco smoke or sociodemographic factors. ${ }^{52}$ The protective mechanism is unknown. However, it is likely to be a combination of maternal behavioral factors, immunological and nutritional properties of human milk, and the physiological influence of sucking on arousal.

Approximately half of SIDS cases occur when infants are sleeping alongside an adult as shown in recent observational case-control studies, with the remainder of deaths occurring among infants who sleep alone in a cot/crib. ${ }^{9,11}$ In a longitudinal study in England of 300 consecutive SIDS deaths for a 20-year period, the total number of bedsharing SIDS deaths decreased by half after the "Back to Sleep" campaign. ${ }^{107}$
However, in this cohort there was a sevenfold reduction in deaths that occurred in the crib/cot, which suggests that placing infants prone to sleep was far more common among those infants sleeping alone than among bedsharing infants. ${ }^{11,107}$ This trend also resulted in a higher proportion of deaths that occurred among bedsharing infants, despite a numerical decrease in bedsharing deaths, because of the lower number of overall deaths. This statistical rise in the proportion of bedsharing deaths led policymakers in some countries to recommend against bedsharing, including the American Academy of Pediatrics, beginning with its 2005 statement. ${ }^{10,108}$ In a meta-analysis of 11 SIDS case-control studies published in 2012, there was a pooled threefold risk associated with bedsharing, although this did not reach significance in older infants ( $>12$ weeks) or those not exposed to tobacco smoke, and the risk was only significant for unintentional bedsharers, not routine bedsharers. In addition, breastfeeding was not included in the analysis. ${ }^{109}$

The interaction between infants bedsharing next to mothers who smoked, as a risk for SIDS, first identified in the New Zealand Cot Death Study in $1993,{ }^{31}$ was more than fourfold (OR: 4.55 [95\% CI: 2.63-7.88]) compared with no risk among infants sleeping next to nonsmoking mothers (OR: 0.98 [95\% CI: 0.44-2.18]) in this case-control study. ${ }^{32} \mathrm{An}$ tenatal smoke exposure is not merely a marker for socioeconomic status as it is associated with reduced infant arousal, and with pathologic findings in the brains of exposed infants. 29,30

A combined analysis of 400 SIDS infants and 1,386 controls from two English studies demonstrated an 18-fold increase in SIDS deaths if either an infant slept with an adult on a sofa or slept next to an adult who drank $>2 \mathrm{U}$ of alcohol within a 24-hour period ( $2 \mathrm{U}$ equals 1 pint or large can of beer [ $440 \mathrm{~mL}], 1$ glass of wine $[175 \mathrm{~mL}]$, or 2 shots of spirits $[50 \mathrm{~mL}])$, with a 4-fold risk with bedsharing if parents smoked. ${ }^{11}$ In the absence of hazards, there was no risk of SIDS with bedsharing compared with nonbedsharers 
(room-sharing or solitary sleeping) (OR: 1.08 [95\% CI: $0.58-2.01])$. When the data were divided into younger $(<3$ months) or older infants, an increased but not statistically significant risk in the younger infants (OR: 1.6 [95\% CI: 0.96-2.7]) and a significant protective effect for nonhazardous bedsharing among the older infants (OR: 0.08 [95\% CI: 0.01-0.52]) were apparent. Further study with larger number of infants would be needed to properly assess any differential effect by infant age or impact of other factors such as parental drug use, infant sleep positioning, or roomsharing.

In contrast, in a similar combined analysis, a fivefold increased risk was associated with younger infants bedsharing in nonhazardous circumstances. ${ }^{9}$ However, the reference group for this study was female breastfed infants placed on their backs next to the beds of nonsmoking parents in the absence of any other risk factors. This magnified the risk difference and renders this explanation not generalizable and difficult to interpret, because both protective factors (detailed in the reference group) and potential risk factors (bedsharing) are being quantified at the same time.

Limited data exist on the risk of bedsharing with caregivers other than the mother. A single study from inner city Chicago in the United States found an increased death risk with multiple bedsharers (other children alone or other children with one or both parents) and nonparent bedsharers, ${ }^{50}$ but a causal relationship is unclear. In a Scottish study, a markedly increased risk of death was found if the infant was sleeping between two parents, ${ }^{51}$ but this study did not account for alcohol and/or drug use, which is notable as the United Kingdom has a high prevalence of heavy episodic drinking (27.1\% among those aged 15 years and older) compared with other industrialized countries. ${ }^{110}$

\section{SIDS epidemiology}

SIDS is most common among low-income ${ }^{46,107}$ and some marginalized communities in wealthy countries, with the world's highest prevalence of SIDS occurring among U.S.

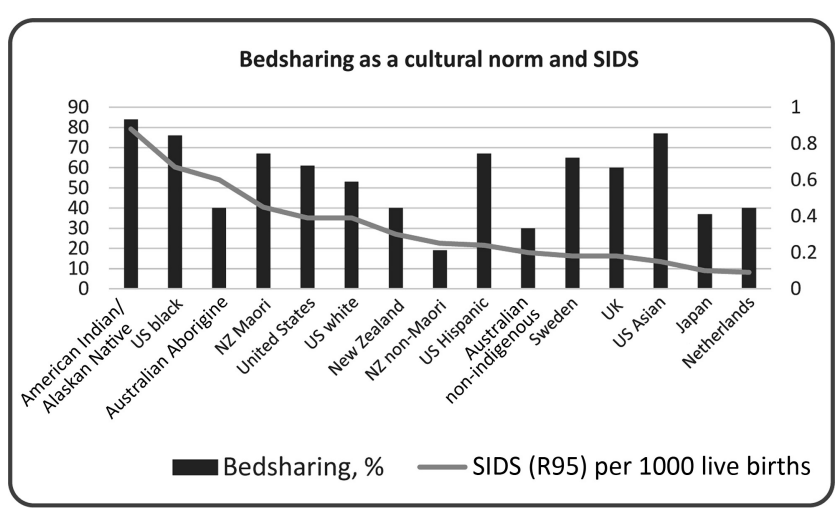

FIG. 4. Bedsharing (any) as a cultural norm and SIDS. Source: Taken from data from Bartick and Tomori, 2019. Most SIDS data are 2014. Australia groups are 2008-2012, Japan is 2015, The Netherlands and Sweden are 2013. Aborigine here refers to both Australian Aborigines and Torres Strait Islanders (combined). SIDS, sudden infant death syndrome.
American Indians/Alaskan Natives (combined) and nonHispanic blacks, New Zealand Māori, Australian Aboriginal and Torres Strait Islander peoples, and indigenous Canadians. ${ }^{33}$ Bedsharing is often common and culturally valued in these marginalized communities. However, there are also many populations with high bedsharing rates that have low rates of SIDS, ${ }^{33,111}$ including Swedes, ${ }^{33}$ U.S. Asians, and U.S. Hispanics (Fig. 4). These conflicting observations may be explained by the presence or absence of a variety of attendant hazardous risk factors. ${ }^{33}$ The overlap of many of the hazardous circumstances with conditions of poverty, structural racism, and legacies of historical trauma must be noted, including antenatal smoking, alcohol use, preterm birth, poor prenatal care, and lack of breastfeeding (feeding human milk substitutes). In the United States, fewer than half of the mothers of infants who died of SIDS received timely prenatal care, ${ }^{33}$ which has been demonstrated as associated with SIDS elsewhere. ${ }^{112,113}$ Structural racism also plays a role. For example, African American infants are more likely to be given human milk substitutes in the hospital without a medical indication, ${ }^{38,39}$ undermining breastfeeding. Racial discrimination of Māori and Australian Indigenous peoples and other minorities by health professionals and society is associated with a wide range of negative health outcomes in New Zealand and Australia. ${ }^{40,114}$

\section{Risk minimization policies and strategies}

Various policies have been adopted to advise parents about bedsharing over the past decade. Countries including the United States, Canada, and Germany ${ }^{73}$ have opted to advise against bedsharing. The conclusion of a 2014 review of all international case-control studies for 20 years by the independent U.K. body, the National Institute for Health and Care Excellence, was that bedsharing in itself is not causal for SIDS, and that parents should be informed of the specific hazards associated with this practice. ${ }^{115}$ In contrast to the countries advising against all bedsharing, countries such as the United Kingdom and Australia ${ }^{116}$ acknowledge that bedsharing occurs both intentionally and unintentionally and is often linked to breastfeeding. Thus, they advise health practitioners to openly discuss the particular circumstances when it would be risky to bedshare.

Although the United Kingdom and Australian approach lacks the simplicity of the predominant U.S. approach, it is more closely aligned to the evidence, which acknowledges that bedsharing is widespread and may be culturally valued. This strategy allows the issue to be discussed without judgment, and for specific hazardous situations to be emphasized. ${ }^{48,117,118}$ The U.S. policy, while calling for nonjudgmental conversations with families about sleep practices, includes the recommendation to room-share without bedsharing. This is a clear direct message to the public that may appear easy to convey to policymakers. A disadvantage of this direct approach, as evidenced by the antibedsharing rhetoric in some campaigns, is the stigmatization that can prohibit honest discussion among parents and health professionals and offend bereaved parents who have lost an infant while bedsharing. Despite campaigns to decrease bedsharing, reported bedsharing has increased in the United States in recent years, especially among black and Hispanic communities. ${ }^{87,91}$ In one trial using enhanced messaging with high-risk families 
FIG. 5. U.S. trends in SIDS and SUID (1990-2015). Source: Centers for Disease Control and Prevention/ National Center for Health Statistics (CDC/NCHS), National Vital Statistics System, Compressed Mortality File. SUID, sudden unexpected infant death.

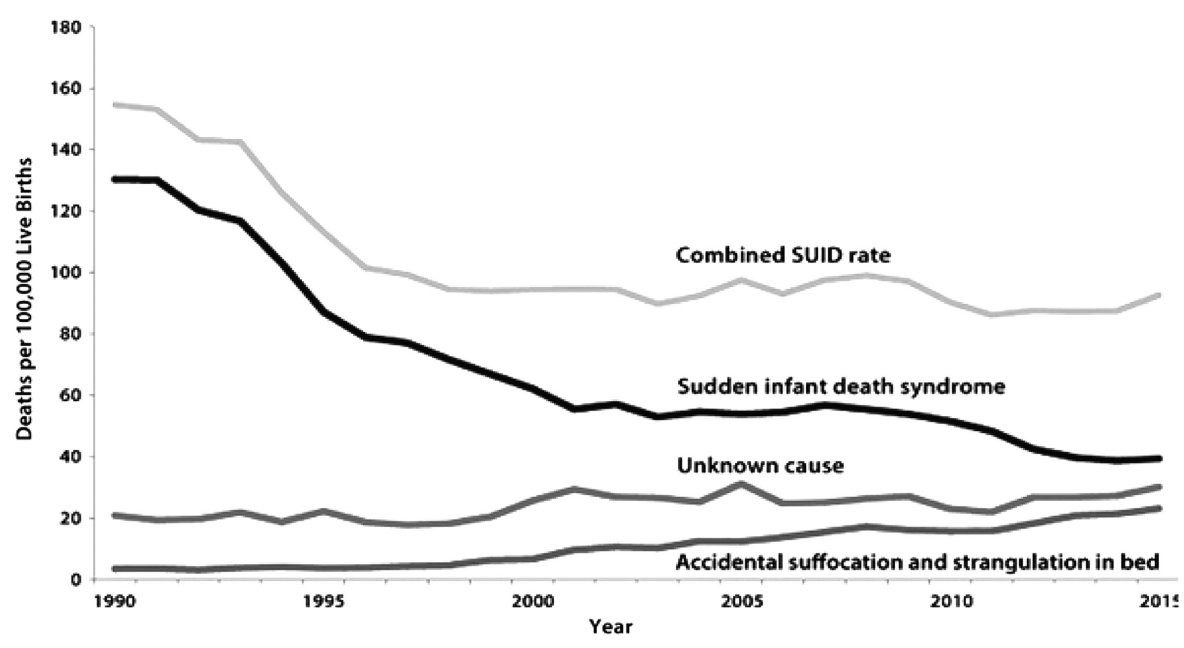

to avoid bedsharing, ${ }^{119}$ bedsharing was no different than in the control group, and in both arms of the study, bedsharing increased over the first 6 months. Breastfeeding did not decrease, presumably because bedsharing was unaffected. ${ }^{22}$ Taking into account diagnostic shift, the proxy SIDS rate $(\mathrm{R} 95+\mathrm{R} 99+\mathrm{W} 75)$ in the United States with the strict "no bedsharing" policy appears to be almost unchanged (Fig. 5). In the United Kingdom, where parents are educated about safe bedsharing, the proxy SIDS rate has fallen over the past 10 years (Fig. 6). It is noted, however, that many different factors other than bedsharing may feed into these recent trends.

Risk minimization strategies include the recognition of the role of breastfeeding in SIDS prevention, the potential risk of bedsharing in the context of hazardous circumstances, and taking into account the importance of the partner and other support persons' involvement in sleep time activities (See Table 3).

\section{Effectiveness of safe sleep interventions}

A review of safe sleep interventions shows that most (primarily U.S.-based) one-on-one interventions are unsuccessful, including those addressing smoke exposure and sleep loca- tion. ${ }^{120}$ One observational U.S. study showed that exclusively breastfeeding women were far less likely to adhere to advice not to bedshare than other women $(65 \%$ versus $30.5 \%) .^{103}$ There has been some success reaching parents with text messages and e-mail with video, social networks, or other media, ${ }^{34,53,54,121}$ but this requires mobile phone and internet access. A simplistic "ABC-Alone, Back, Crib" approach is often rejected by families and caregivers, and has not been shown to decrease sleep-related deaths, leading to the adoption of a conversational approach, ${ }^{35}$ which includes exploring patients' needs and perceptions and making informed decisions. ${ }^{118}$ Annual U.S. surveys between 1993 and 2010 revealed that bedsharing rates among black and Hispanic families has continually increased, despite nearly half of U.S. nighttime caregivers discussing bedsharing with a physician. ${ }^{91}$

New Zealand, whose Māori population until recently had the highest SIDS rate in the world, driven by high rates of smoking combined with bedsharing, ${ }^{58}$ has seen reductions in infant death rates after a novel intervention. ${ }^{122}$ It targets high-risk families with provision of the wahakura (Fig. 3c), a woven flax bassinet-like structure based on Māori traditions developed for this purpose, used on the bed, to create a separate sleeping space, and the Pépi-Pod ${ }^{\mathrm{TM}}$ (Fig. 3d), a polypropylene
FIG. 6. England and Wales trends in SIDS and SUID (20002015). Source: Office for National Statistics, England and Wales. SUDI, sudden unexpected death in infancy.

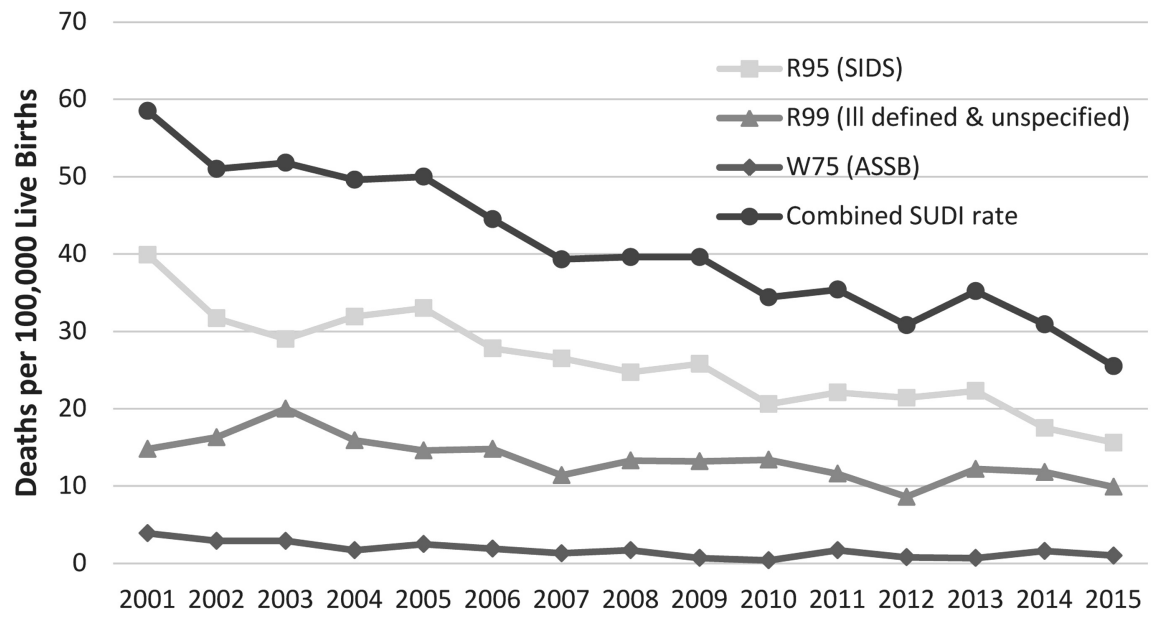


box (food-grade plastic) of similar proportions. ${ }^{123}$ Parents also receive one-on-one advice that promotes "Safe Sleep." Importantly, the wahakura has the enhanced appeal of reclaiming a traditional Māori infant care practice that supports the highly valued proximity of mother and infant. ${ }^{124}$ The high Māori SIDS rate dropped dramatically between 2009 and 2012, especially in targeted areas. ${ }^{122}$ Interestingly, the wahakura did not decrease unsafe sleep behaviors compared with a bedside bassinet, but was associated with markedly increased breastfeeding rates at 6 months (wahakura: $22.5 \%$ versus bassinet $10.7 \%, p=0.04){ }^{55}$

\section{Future areas of research}

- How can death investigation techniques be improved to determine whether the death of an infant can be fully explained by asphyxia?

- Is there a significant risk from bedsharing in the absence of all hazardous circumstances?

- Is there a relationship between bedsharing risk and infant age and mode of feeding (adjusting for all hazardous circumstances) (breastfeeding, expressed milk feeding, donor milk feeding, and human milk substitutes)?

- What is the best advice for a safe sleep environment for nonbreastfeeding infants?

- How do multiple bedsharers in the absence of hazardous risks, including bedsharing of twins, impact the safety of the infant? Does the location of the infant in the bed, for example, between the parents or on the edge of the bed next to the mother, make a difference for safety?

- Are in-bed devices and sidecars safe and efficacious, especially for infants in high-risk situations, and if safe, what are their effects on breastfeeding?

- Can the C-position ("cuddle curl") be adopted by nonbreastfeeding bedsharers and is it effective in reducing SIDS?

- Is providing supportive information to parents on "breastsleeping," as defined in this protocol as a separate category of bedsharing, protective or risky to provide these parents with better specific guidance?

- Is there autopsy evidence of the effect of feeding human milk substitutes that can establish a causal link between feeding human milk substitutes and SIDS?

$\circ$ Do the risks of death from SIDS due to early weaning and potentially related to the lack of safe bedsharing outweigh the risk of death from SIDS from bedsharing in nonhazardous circumstances? There are many confounders and this will be difficult to study.

- To what extent does maternal obesity modify the risks and benefits of bedsharing? ${ }^{125,126}$

\section{Disclosure Statement}

J.J.M. serves as safety consultant for the Arms Reach cosleeper for which he is paid a small honorarium but no royalties. No other competing financial interests exist. P.S.B. serves as the chair of the International Society for the Study and Prevention of Perinatal and Infant Death (ISPID) and is a scientific advisor to UNICEF UK, neither of which are funded positions. H.L.B. is scientific advisor to Lullaby Trust and chair of the Lullaby Trust Scientific Committee, board member of ISPID, and member of editorial review board for Journal of Human Lactation, which are all unfunded positions. As part of her academic role, she is co-founder and co-director of Basis, the Baby Sleep Information Source (www.BasisOnline.org.uk). J.J.M. serves as psychological advisor to Fit Pregnancy magazine, an unpaid respondent for the website Kids in the House, KidsintheHouse.com, and a board member of Speaking of Kids; L.F.W. works as a consultant and physician lead for Communities and Hospitals Advancing Maternity Practices (CHAMPS), Boston Medical Center, Boston, Massachusetts, and as a consultant to National Institute for Children's Health Quality (NICHQ)-National Action Partnership to Promote Safe Sleep Improvement and Innovation Network (NAPPSS-IIN). She is chair of the Section on Breastfeeding for the American Academy of Pediatrics (AAP) and also serves on the AAP Task Force on SIDS.* K.A.M. serves on the clinical advisory board of the US BabyFriendly Hospital Initiative and is associate editor of the Journal of Human Lactation; M.C.B. has received funding from the W.K. Kellogg Foundation for research in breastfeeding and economics and co-leads the Massachusetts Baby-Friendly Collaborative. L.F.W. and M.C.B. serve on the board of the Academy of Breastfeeding Medicine.

*Correction added on July 30, 2021 after first online publication of January 3, 2020: This article reflects the personal opinion of Dr. Feldman-Winter and does not represent AAP policy or guidelines.

\section{Funding Information}

This work was unfunded.

\section{References}

1. Ball HL, Ward-Platt MP, Heslop E, et al. Randomised trial of infant sleep location on the postnatal ward. Arch Dis Child 2006;91:1005-1010.

2. Huang Y, Hauck FR, Signore C, et al. Influence of bedsharing activity on breastfeeding duration among US mothers. JAMA Pediatr 2013;167:1038-1044.

3. Blair PS, Heron J, Fleming PJ. Relationship between bed sharing and breastfeeding: Longitudinal, population-based analysis. Pediatrics 2010;126: 1119-1126.

4. Ball HL, Howel D, Bryant A, et al. Bed-sharing by breastfeeding mothers: Who bed-shares and what is the relationship with breastfeeding duration? Acta Paediatr 2016;105:628-634.

5. Ball HL. Night-time infant care: Cultural practice, evolution, and infant devopment. In: Childrearing and Infant Care Issues: A Cross-Cultural Perspective, Liamputtong P, ed. Melbourne, Australia: Nova Science, 2006.

6. McKenna J, Mosko S, Richard C. Bedsharing promotes breastfeeding. Pediatrics 1997;100:214-219.

7. Moon RY, Task Force on Sudden Infant Death Syndrome. SIDS and other sleep-related infant deaths: Evidence base for 2016 updated recommendations for a safe infant sleeping environment. Pediatrics 2016;138:e20162940.

8. Ball HL. Breastfeeding, bed-sharing, and infant sleep. Birth 2003;30:181-188.

9. Carpenter R, McGarvey C, Mitchell EA, et al. Bed sharing when parents do not smoke: Is there a risk of SIDS? An 
individual level analysis of five major case-control studies. BMJ Open 2013;3:e02299.

10. Task Force on Sudden Infant Death Syndrome. SIDS and other sleep-related infant deaths: Updated 2016 recommendations for a safe infant sleeping environment. Pediatrics 2016;138:e20162938.

11. Blair PS, Sidebotham P, Pease A, et al. Bed-sharing in the absence of hazardous circumstances: Is there a risk of sudden infant death syndrome? An analysis from two case-control studies conducted in the UK. PLoS One 2014;9:e107799.

12. Victora CG, Bahl R, Barros AJ, et al. Breastfeeding in the 21st century: Epidemiology, mechanisms, and lifelong effect. Lancet 2016;387:475-490.

13. Howick J, Chalmers I, Glasziou P, et al. The Oxford 2011 Levels of Evidence. UK: Oxford, 2011.

14. Bovbjerg ML, Hill JA, Uphoff AE, et al. Women who bedshare more frequently at 14 weeks postpartum subsequently report;onger durations of breastfeeding. J Midwifery Womens Health 2018;63:418-424.

15. Feldman-Winter L, Goldsmith JP, et al. Safe sleep and skin-to-skin care in the neonatal period for healthy term newborns. Pediatrics 2016;138:e20161889.

16. Lullaby Trust, Baby Sleep Info Source (Basis), Public Health England, UNICEF UK Baby-Friendly Hospital Initiative. Safer sleep for babies: A guide for parents. London, 2019. https://www.unicef.org.uk/babyfriendly/wpcontent/uploads/sites/2/2018/08/Caring-for-your-baby-atnight-web.pdf

17. Crenshaw JT. Healthy Birth Practice \#6: Keep mother and baby together: It's best for mother, baby, and breasfeeding. J Perinatal Edu 2014;23:211-217.

18. Drever-Smith C, Bogossian F, New K. Co-sleeping and bed sharing in postnatal maternity units: A review of the literature and critique of clinical practice guidelines. Int $J$ Childbirth 2013;3:13-27.

19. Fetherston CM, Leach JS. Analysis of the ethical issues in the breastfeeding and bedsharing debate. Breastfeeding Rev 2012;20:7-17.

20. Bajanowski T, Vege A, Byard RW, et al. Sudden infant death syndrome (SIDS) — standardised investigations and classification: Recommendations. Forensic Sci Int 2007; 165:129-143.

21. Baddock SA, Purnell MT, Blair PS, et al. The influence of bed-sharing on infant physiology, breastfeeding and behaviour: A systematic review. Sleep Med Rev 2019;43:106-117.

22. Moon RY, Mathews A, Joyner BL, et al. Impact of a randomized controlled trial to reduce bedsharing on breastfeeding rates and duration for African-American infants. J Community Health 2017;42:707-715.

23. Sackett DL, Rosenberg WM, Gray JA, et al. Evidence based medicine: What it is and what it isn't. BMJ 1996; 312:71-72.

24. Jack E, Maskrey N, Byng R. SHERPA: A new model for clinical decision making in patients with multimorbidity. Lancet 2018;392:1397-1399.

25. Ball HL. Reasons to bed-share: Why parents sleep with their infants. J Reprod Infant Physiol 2002;20:207-221.

26. Ostfeld BM, Schwartz-Soicher O, Reichman NE, et al. Prematurity and sudden unexpected infant deaths in the United States. Pediatrics 2017;140:e20163334.

27. Malloy MH, Hoffman HJ. Prematurity, sudden infant death syndrome, and age of death. Pediatrics 1995; 96(3 Pt. 1):464-471.
28. Zhang $\mathrm{K}$, Wang $\mathrm{X}$. Maternal smoking and increased risk of sudden infant death syndrome: A meta-analysis. Leg Med (Tokyo) 2013;15:115-121.

29. Lavezzi AM, Mecchia D, Matturri L. Neuropathology of the area postrema in sudden intrauterine and infant death syndromes related to tobacco smoke exposure. Auton Neurosci 2012;166:29-34.

30. Kinney HC, Thach BT. The sudden infant death syndrome. N Engl J Med 2009;361:795-805.

31. Scragg R, Mitchell EA, Taylor BJ, et al. Bed sharing, smoking, and alcohol in the sudden infant death syndrome. New Zealand Cot Death Study Group. BMJ 1993; 307:1312-1318.

32. Mitchell EA, Thompson JM, Zuccollo J, et al. The combination of bed sharing and maternal smoking leads to a greatly increased risk of sudden unexpected death in infancy: The New Zealand SUDI Nationwide Case Control Study. N Z Med J 2017;130:52-64.

33. Bartick M, Tomori C. Sudden infant death and social justice: A syndemics approach. Matern Child Nutr 2019; 15:e12652.

34. Moon RY, Corwin MJ, Kerr S, et al. Mediators of improved adherence to infant safe sleep using a mobile health intervention. Pediatrics 2019;143:e20182799.

35. Bronheim S. Building on Campaigns with Conversations: An Individualized Approach to Helping Families Embrace Safe Sleep and Breastfeeding. Washington, DC: National Center for Education in Maternal and Child Health, 2017.

36. UNICEF UK Baby-Friendly Hospital Initiative. Cosleeping and SIDS: A Guide for Health Professionals. London: UNICEF UK, 2019.

37. Filippidis FT, Laverty AA, Hone T, et al. Association of cigarette price differentials with infant mortality in 23 European Union countries. JAMA Pediatr 2017;171:1100-1106.

38. Lind JN, Perrine CG, Li R, et al. Racial disparities in access to maternity care practices that support breastfeedingUnited States, 2011. MMWR Morb Mortal Wkly Rep 2014; 63:725-728.

39. McKinney CO, Hahn-Holbrook J, Chase-Lansdale PL, et al. Racial and ethnic differences in breastfeeding. $P e$ diatrics 2016;138:e20152388.

40. Harris R, Cormack D, Tobias M, et al. The pervasive effects of racism: Experiences of racial discrimination in New Zealand over time and associations with multiple health domains. Soc Sci Med 2012;74:408-415.

41. Kemp JS, Nelson VE, Thach BT. Physical properties of bedding that may increase risk of sudden infant death syndrome in prone-sleeping infants. Pediatr Res 1994; 36(1 Pt. 1):7-11.

42. Thompson JMD, Tanabe K, Moon RY, et al. Duration of breastfeeding and risk of SIDS: An individual participant data meta-analysis. Pediatrics 2017;140:e20171324.

43. Li DK, Petitti DB, Willinger M, et al. Infant sleeping position and the risk of sudden infant death syndrome in California, 1997-2000. Am J Epidemiol 2003;157:446-455.

44. Tuladhar R, Harding R, Cranage SM, et al. Effects of sleep position, sleep state and age on heart rate responses following provoked arousal in term infants. Early Hum Dev 2003;71:157-169.

45. Torres LH, Balestrin NT, Spelta LEW, et al. Exposure to tobacco smoke during the early postnatal period modifies receptors and enzymes of the endocannabinoid system in the brainstem and striatum in mice. Toxicol Lett 2019;302:35-41. 
46. Erck Lambert AB, Parks SE, Cottengim C, et al. Sleeprelated infant suffocation deaths attributable to soft Bedding, overlay, and wedging. Pediatrics 2019;143:e20183408.

47. Lagon E, Moon RY, Colvin JD. Characteristics of infant deaths during sleep while under nonparental supervision. J Pediatr 2018;197:57.e36-62.e36.

48. UNICEF UK Baby-Friendly Hospital Initiative. Caring for Your Baby at Night. London: UNICEF UK, 2016.

49. Weissinger D, West D, Smith LJ, et al. Sweet Sleep: Nighttime and Naptime Strategies for the Breastfeeding Family. New York: Ballantine Books, 2014.

50. Hauck FR, Herman SM, Donovan M, et al. Sleep environment and the risk of sudden infant death syndrome in an urban population: The Chicago Infant Mortality Study. Pediatrics 2003;111(5 Pt. 2):1207-1214.

51. Tappin D, Ecob R, Brooke H. Bedsharing, roomsharing, and sudden infant death syndrome in Scotland: A casecontrol study. J Pediatr 2005;147:32-37.

52. Hauck FR, Thompson JM, Tanabe KO, et al. Breastfeeding and reduced risk of sudden infant death syndrome: A meta-analysis. Pediatrics 2011;128:103-110.

53. Kellams A, Parker MG, Geller NL, et al. TodaysBaby Quality Improvement: Safe Sleep Teaching and Role Modeling in 8 US Maternity Units. Pediatrics 2017;140:e20171816.

54. Moon RY, Hauck FR, Kellams AL, et al. Comparison of text messages versus e-mail when communicating and querying with mothers about safeiInfant sleep. Acad Pediatr 2017;17:871-878.

55. Baddock SA, Tipene-Leach D, Williams SM, et al. Wahakura versus bassinet for safe infant sleep: A randomized trial. Pediatrics 2017;139:e20160162.

56. World Health Organization. ICD-10, International Statistical Classification of Diseases and Related Health Problems-10th Revision, 5th edition. Geneva, Switzerland: WHO, 2018.

57. Goldstein RD, Blair PS, Sens MA, et al. Inconsistent classification of unexplained sudden deaths in infants and children hinders surveillance, prevention and research: Recommendations from The 3rd International Congress on Sudden Infant and Child Death. Forensic Sci Med Pathol 2019;4:622-628.

58. Taylor BJ, Garstang J, Engelberts A, et al. International comparison of sudden unexpected death in infancy rates using a newly proposed set of cause-of-death codes. Arch Dis Child 2015;100:1018-1023.

59. Shapiro-Mendoza CK, Parks SE, Brustrom J, et al. Variations in cause-of-death determination for sudden unexpected infant deaths. Pediatrics 2017;140:e20170087.

60. Marinelli KA, Ball HL, McKenna JJ, et al. An integrated analysis of maternal-infant sleep, breastfeeding, and sudden infant death syndrome: Research supporting a balanced discourse. J Hum Lact 2019;35:510-520.

61. Hernell O. Human milk vs. cow's milk and the evolution of infant formulas. Nestle Nutr Workshop Ser Pediatr Program 2011;67:17-28.

62. Casiday RE, Wright CM, Panter-Brick C, et al. Do early infant feeding patterns relate to breast-feeding continuation and weight gain? Data from a longitudinal cohort study. Eur J Clin Nutr 2004;58:1290-1296.

63. Ball HL. Evolution-informed maternal-infant health. Nat Ecol Evol 2017;1:73.

64. McKenna JJ, Gettler LT. There is no such thing as infant sleep, there is no such thing as breastfeeding, there is only breastsleeping. Acta Paediatr 2015;105:17-21.
65. Ball HL. Evolutionary paediatrics: A case study in applying Darwinian medicine. In: Medicine and Evolution: Current Applications, Future Prospects, Vol. 48, Elton S, O’Higgins P, eds. Boca Raton, FL: Taylor \& Francis, 2008, pp. 127-152.

66. McKenna JJ, Ball HL, Gettler JT. Mother-infant cosleeping, breastfeeding and sudden infant death syndrome: What biological anthropology has discovered about normal infant sleep and pediatric sleep medicine. Am J Phys Anthropol 2007(Suppl. 45):133-161.

67. Trevathan WR, Rosenberg KR. Human evolution and the helpless infant. In: Costly and Cute: Helpless Infants and Human Evolution, Trevathan WR, ed. Albuquerque: University of New Mexico Press, 2015, pp. 1-28.

68. Stearns PN, Rowland P, Giarnella L. Children's sleep: Sketching historical change. J Soc Hist 1996;30:345-366.

69. Centers for Disease Control and Prevention. Infant Feeding Practices Study II, Chapter 3, Infant Feeding. Atlanta, GA: CDC, 2008.

70. Kahn A, Groswasser J, Franco P, et al. Factors influencing the determination of arousal thresholds in infants-A review. Sleep Med 2000;1:273-278.

71. Canadian Paediatric Society, Canadian Foundation for the Study of Infant Deaths, Canadian Institute of Child Health, Health Canada, Public Health Agency of Canada. Joint Statement on Safe Sleep: Preventing Sudden Infant Deaths in Canada. Ottawa, Canada: Canadian Paediatric Society, 2018.

72. European Foundation for the Care of Newborn Infants. Safe Sleep. Munich, Germany: EFCNI, 2018.

73. Kindergesundheit-info.de [Childhealth-info]. Ein schmerzliches Thema: Der Plötzliche Kindstod. Bundeszentrale für gesundheitliche Aufklärfung. [A Painful Topic: Sudden Infant Death Syndrome. Federal Center for Health Education]. Published 2019. Available at https://www .kindergesundheit-info.de/themen/risiken-vorbeugen/ ploetzlicher-kindstod-sids/sids (accessed September 13, 2019).

74. Tomori C. Breastsleeping in four cultures: Comparative analysis of a biocultural body technique. In: Breastfeeding: New Anthropological Approaches, Tomori C, Palmquist AE, Quinn E, eds. Abington, NY: Routledge, 2017, pp. 55-68.

75. Ball HL, Russell CK. Nighttime nurturing: An evolutionary perspective on breastfeeding and sleep. In: Evolution, Early Experience and Human Development: From Research to Practice and Policy, Narvaez D, Panksepp J, Schore A, Gleason T, eds. Oxford: Oxford University Press, 2012, pp. 241-261.

76. Ball HL, Klingaman K. Breastfeeding and mother-infant sleep proximity: Implications for infant care. In: Evolutionary Medicine and Health: New Perspectives, Trevathan WR, McKenna JJ, eds. New York: Oxford University Press, 2008, pp. 226-241.

77. Mobbs EJ, Mobbs GA, Mobbs AE. Imprinting, latchment and displacement: A mini review of early instinctual behaviour in newborn infants influencing breastfeeding success. Acta Paediatr 2016;105:24-30.

78. Ball HL. Parent-infant bed-sharing behavior: Effects of feeding type and presence of father. Hum Nat 2006;17:301-318.

79. Mosko S, Richard C, McKenna J. Maternal sleep and arousals during bedsharing with infants. Sleep 1997;20: 142-150.

80. Mosko S, Richard C, McKenna J. Infant arousals during mother-infant bed sharing: Implications for infant sleep 
and sudden infant death syndrome research. Pediatrics 1997;100:841-849.

81. Volpe LE, Ball HL, McKenna JJ. Nighttime parenting strategies and sleep-related risks to infants. Soc Sci Med 2013;79:92-100.

82. Lester BM, Conradt E, LaGasse LL, et al. Epigenetic programming by maternal behavior in the human infant. Pediatrics 2018;142:e20180194.

83. Blair PS, Ball HL. The prevalence and characteristics associated with parent-infant bed-sharing in England. Arch Dis Child 2004;89:1106-1110.

84. McCoy RC, Hunt CE, Lesko SM, et al. Frequency of bed sharing and its relationship to breastfeeding. J Dev Behav Pediatr 2004;25:141-149.

85. Gibson E, Dembofsky CA, Rubin S, et al. Infant sleep position practices 2 years into the "back to sleep" campaign. Clin Pediatr (Phila) 2000;39:285-289.

86. Rigda RS, McMillen IC, Buckley P. Bed sharing patterns in a cohort of Australian infants during the first six months after birth. J Paediatr Child Health 2000;36:117-121.

87. Bombard JM, Kortsmit K, Warner L, et al. Vital Signs: Trends and disparities in infant safe sleep practices United States, 2009-2015. MMWR Morb Mortal Wkly Rep 2018;67:39-46.

88. Hauck FR, Tanabe KO. International trends in sudden infant death syndrome: Stabilization of rates requires further action. Pediatrics 2008;122:660-666.

89. Ateah CA, Hamelin KJ. Maternal bedsharing practices, experiences, and awareness of risks. J Obstet Gynecol Neonatal Nurs 2008;37:274-281.

90. Santos IS, Mota DM, Matijasevich A, et al. Bed-sharing at 3 months and breast-feeding at 1 year in southern Brazil. J Pediatr 2009;155:505-509.

91. Colson ER, Willinger M, Rybin D, et al. Trends and factors associated with infant bed sharing, 1993-2010: The National Infant Sleep Position Study. JAMA Pediatr 2013; 167:1032-1037.

92. Salm Ward TC. Reasons for mother-infant bed-sharing: A systematic narrative synthesis of the literature and implications for future research. Matern Child Health J 2015; 19:675-690.

93. Crane D, Ball HL. A qualitative study in parental perceptions and understanding of SIDS-reduction guidance in a UK bi-cultural urban community. BMC Pediatr 2016;16:23.

94. Culver ED. Exploring bed-sharing mothers' motives and decision-making for getting through the night intact: A grounded theory. J Midwifery Womens Health 2009;54: 423.

95. Rudzik AEF, Ball HL. Exploring maternal perceptions of infant sleep and feeding method among mothers in the United Kingdom: A qualitative focus group study. Matern Child Health J 2016;20:33-40.

96. Volpe LE, Ball HL. Infant sleep-related deaths: Why do parents take risks? Arch Dis Child 2015;100:603-604.

97. Joyner BL, Oden RP, Ajao TI, et al. Where should my baby sleep: A qualitative study of African American infant sleep location decisions. J Natl Med Assoc 2010;102:881-889.

98. Chianese J, Ploof D, Trovato C, et al. Inner-city caregivers' perspectives on bed sharing with their infants. Acad Pediatr 2009;9:26-32.

99. McKenna JJ, Volpe LE. Sleeping with baby: An internet based sampling of parental experiences, choices, perceptions, and interpretations in a Western industrialized context. Infant Child Dev 2007;16:359-385.
100. Tomori C. Nighttime Breastfeeding: An American Cultural Dilemma. New York: Berghahn Books, 2014.

101. Quillin SI, Glenn LL. Interaction between feeding method and co-sleeping on maternal-newborn sleep. J Obstet Gynecol Neonatal Nurs 2004;33:580-588.

102. Tully KP, Ball HL. Trade-offs underlying maternal breastfeeding decisions: A conceptual model. Matern Child Nutr 2013;9:90-98.

103. Smith LA, Geller NL, Kellams AL, et al. Infant sleep location and breastfeeding practices in the United States, 2011-2014. Acad Pediatr 2016;16:540-549.

104. Bartick M, Tomori C, Ball HL. Babies in boxes and the missing links on safe sleep: Human evolution and cultural revolution. Matern Child Nutr 2018;14:e12544.

105. Bartick M, Smith LJ. Speaking out on safe sleep: Evidence-based infant sleep recommendations. Breastfeed Med 2014;9:417-422.

106. Kendall-Tackett K, Cong Z, et al. Mother-infant sleep locations and nighttime feeding behavior: U.S. data from the survey of mothers' sleep and fatigue. Clin Lact 2010;1(Fall).

107. Blair PS, Sidebotham P, Berry PJ, et al. Major epidemiological changes in sudden infant death syndrome: A 20year population-based study in the UK. Lancet 2006;367: 314-319.

108. American Academy of Pediatrics Task Force on Sudden Infant Death Syndrome. The changing concept of sudden infant death syndrome: Diagnostic coding shifts, controversies regarding the sleeping environment, and new variables to consider in reducing risk. Pediatrics 2005;116:1245-1255.

109. Vennemann MM, Hense HW, Bajanowski T, et al. Bed sharing and the risk of sudden infant death syndrome: Can we resolve the debate? J Pediatr 2012;160:44.e42-48.e42.

110. World Health Organization. Global Status Report on Alcohol and Health 2014. Geneva, Switzerland: WHO, 2014.

111. McKenna JJ, McDade T. Why babies should never sleep alone: A review of the co-sleeping controversy in relation to SIDS, bedsharing and breast feeding. Paediatr Respir Rev 2005;6:134-152.

112. Kohlendorfer U, Haberlandt E, Kiechl S, et al. Pre- and postnatal medical care and risk of sudden infant death syndrome. Acta Paediatr 1997;86:600-603.

113. Mitchell EA, Scragg R, Stewart AW, et al. Results from the first year of the New Zealand cot death study. N Z Med J 1991;104:71-76.

114. Shepherd CCJ, Li J, Cooper MN, Hopkins KD, et al. The impact of racial discrimination on the health of Australian Indigenous children aged 5-10 years: Analysis of national longitudinal data. Int J Equity Health 2017;16:116.

115. NICE (National Institute for Health Care Excellence). Appendix A CG37: Summary of New Evidence from Surveillance, Post-natal Care Up to 8 Weeks After Birth. London: NICE, 2015.

116. Red Nose National Scientific Advisory Group. Information statement: Sharing a sleep surface with a baby. Published 2018. Available at https://rednose.org.au/article/sharing-asleep-surface-with-a-baby (accessed September 13, 2019).

117. Ball HL. The Atlantic Divide: Contrasting U.K. and U.S. recommendations on cosleeping and bed-sharing. J Hum Lact 2017;33:765-769.

118. Young J, Shipstone R. Shared sleeping surfaces and dangerous sleeping environments. In: SUDS Sudden Infant and Early Childhood Death: The Past, the Present and the Future, Duncan JR, Byard JR, eds. Adelaide, Australia: University of Adelaide Press, 2018. 
119. Moon RY, Mathews A, Joyner BL, et al. Health messaging and African-American infant sleep location: A randomized controlled trial. J Community Health 2017;42:1-9.

120. Salm Ward TC, Balfour GM. Infant safe sleep interventions, 1990-2015: A review. J Community Health 2016; 41:180-196.

121. Moon RY, Mathews A, Oden R, et al. A qualitative analysis of how mothers' social networks are established and used to make infant care decisions. Clin Pediatr (Phila) 2019;58:985-992.

122. Mitchell EA, Cowan S, Tipene-Leach D. The recent fall in postperinatal mortality in New Zealand and the Safe Sleep programme. Acta Paediatr 2016;105:1312-1320.

123. Abel S, Tipene-Leach D. SUDI prevention: A review of Maori safe sleep innovations for infants. $N Z$ Med $J$ 2013; 126:86-94.

124. Abel S, Stockdale-Frost A, Rolls R, et al. The wahakura: A qualitative study of the flax bassinet as a sleep location for New Zealand Maori infants. N Z Med J 2015;128: 12-19.

125. Mitchell EA, Thompson JMD. Who cosleeps? Does high maternal body weight and duvet use increase the risk of sudden infant death syndrome when bedsharing? Paediatr Child Health 2006;11:14A-15A.

126. Carroll-Pankhurst C, Mortimer EA, Jr. Sudden infant death syndrome, bedsharing, parental weight, and age at death. Pediatrics 2001;107:530-536.

$\mathrm{ABM}$ protocols expire 5 years from the date of publication. Content of this protocol is up-to-date at the time of publication. Evidence-based revisions are made within 5 years or sooner if there are significant changes in the evidence.
Peter S. Blair, PhD

Helen L. Ball, PhD

James J. McKenna, PhD

Lori Feldman-Winter, MD, MPH

Kathleen A. Marinelli, MD, FABM

Melissa C. Bartick, MD, FABM

The Academy of Breastfeeding Medicine Protocol

Committee Members 2020:

Michal Young, MD, FABM, Chairperson

Larry Noble, MD, FABM, Translations Chairperson

Sarah Calhoun, MD

Megan Elliott-Rudder, MD

Laura Rachael Kair, MD, FABM

Susan Lappin, $M D$

Ilse Larson, $M D$

Ruth A. Lawrence, MD, FABM

Yvonne Lefort, MD, FABM

Nicole Marshall, MD, MCR

Katrina Mitchell, MD, FABM

Catherine Murak, MD

Eliza Myers, $M D$

Sarah Reece-Stremtan, $M D$

Casey Rosen-Carole, MD, MPH, MSEd

Susan Rothenberg, MD, FABM

Tricia Schmidt, $M D$

Tomoko Seo, MD, FABM

Natasha Sriraman, MD

Elizabeth K. Stehel, $M D$

Nancy Wight, MD

Adora Wonodi, MD

For correspondence: abm@bfmed.org 